\title{
Elevation, pitch and travel axis stabilization of 3DOF helicopter with hybrid control system by GA-LQR based PID controller
}

\author{
Ibrahim K. Mohammed, Abdulla I. Abdulla \\ Department of Systems and Control Engineering, College of Electronics Engineering, Ninevah University, Iraq
}

\begin{tabular}{l}
\hline \hline Article Info \\
\hline Article history: \\
Received Apr 29, 2019 \\
Revised Aug 28, 2019 \\
Accepted Oct 9, 2019 \\
\hline
\end{tabular}

Keywords:

3DOF helicopter system

Genetic algorithm

Hybrid control

LQR controller

PID controller

\begin{abstract}
This research work introduces an efficient hybrid control methodology through combining the traditional proportional-integral-derivative (PID) controller and linear quadratic regulator (LQR) optimal controlher. The proposed hybrid control approach is adopted to design three degree of freedom (3DOF) stabilizing system for helicopter. The gain parameters of the classic PID controller are determined using the elements of the LQR feedback gain matrix. The dynamic behaviour of the LQR based PID controller, is modeled in state space form to enable utlizing state feedback controller technique. The performance of the proposed LQR based LQR controller is improved by using Genetic Algorithm optimization method which are adopted to obtain optimum values for $L Q R$ controller gain parameters. The LQR-PID hybrid controller is simulated using Matlab environment and its performance is evaluated based on rise time, settling time, overshoot and steady state error parameters to validate the proposed $3 \mathrm{DOF}$ helicopter balancing system. Based on GA tuning approach, the simulation results suggest that the hybrid LQR-PID controller can be effectively employed to stabilize the 3DOF helicopter system.
\end{abstract}

Copyright $\left({ }_{0} 2020\right.$ Institute of Advanced Engineering and Science. All rights reserved.

\section{Corresponding Author:}

Ibrahim K. Mohammed,

Department of Systems and Control Engineering,

College of Electronics Engineeing,

Ninevah University, Mosul, Iraq

Email: ibrahim.mohammed@uoninevah.edu.iq

\section{INTRODUCTION}

The A 3DOF helicopter model is a multi-input, multi-output system (MIMO) with strongly nonlinear characteristics, used as experimental platform for verification of various control algorithms. The helicopter is a complex mechanical scheme and has unstable dynamics which make using traditional control methods to stabilize it is a big challenge. In control procedure of 3DOF helicopter system the nonlinear dynamics is linearised about preselected operating conditions. Based on linearised system model a classical proportional integral derivative PID controller technique is widely used due to its simplicity and easy to realize [1]. However, using conventional PID technique is insufficient to guide the helicopter through the demand trajectory as the regulation of the controller parameters is hard task even for an experienced control engineers [1, 2]. In another control method, an optimal tracking approach uilizing LQR controller to stabilize the helicopter system was proposed by [3]. In the proposed system, a Genetic Algorithm (GA) is employed to optimize a classic LQR controller for position control of DC motor. By using LQR controller a significant improvement in guidance of helicopter system is carried out. Further investigations in control strategies for helicopter system are still the goal of many researchers. A new control approach based on hybrid controller technique was proposed by many researchers to control the 3DOF helicopter system. Fan and Joo [4] proposed a hybrid control method using fuzzy and PID controller to balance the 3DOF helicopter system at desired equilibrium position. In this study, an efficient 
control method has been adopted to propose a hybrid control strategy using both conventional and intelligent control algorithms. This hybrid control approach uses PID controller as a conventional control, while the liner quadratic regulator (LQR) controller based on GA used as an intelligent control. The LQR optimal controller is recommended to implement the intelligent system due to its high precision in movement applications [5]. In this state feedback controller technique a tradeoff between the plant characteristics and the input effort of the system can be achieved if the controller gain parameters are tuned propperly [6-8].

The LQR controller design can be improved by employing soft computing and optimization techniques, which are adopted to achieve tuning proecss of the controller gain parameters, such as Differential Evolution (DE) [9], Particle Swarm Inspired Evolutionary Algorithm (PS-EA) [10], Particle Swarm Optimisation (PSO) [11], Genetic Algorithm (GA) [12], memetic algorithm (MA) [13], Imperialist Competitive Algorithm (ICA) [14], Ant Colony Optimization (ACO) [15], Artificial Bee Colony (ABC) [16], and Artificial Immune Systems (AIS) [17]. In order to validate the proposed helicopter model, the hybrid control system is simulated by using the Matlab environment, and its balancing performance is then evaluated based on stability criteria parameters which include rise and settling time, overshoot and control input. The purpose of the proposed hybrid GA-LQR based PID controller is to design an efficient control system utilized to stabilize a prototype 3DOF helicopter system at desired roll and pitch positions as well as angular travel speed. During the current decade there has been a considerable interest by many control researchers in using of hybrid control approach in various engineering applications. Vendoh and Jovitha [18] presented a hybrid control system using LQR based PID controller for stabilization and trajectory tracking of magnetic levitation system. Arbab et al. [19] proposed a hybrid control strategy using fuzzy based LQR controller for 3-DOF helicopter system. Choudhary [1] design another hybrid control scheme using LQR based PID controller for 3-DOF helicopter system, however, the introduced hybrid control systems are not efficient as the parameters of the LQR feedback gain matrix are obtained by applying trial and error procedure. The paper presents a simple method for the approximation of PID controller gain parameters from feedback gain matrix of the controller. The LQR controller gain is obtained from the weighting matrices $\mathrm{Q}$ and $\mathrm{R}$ which their elements values are tuned effectively by the GA optimization method.

The rest of the paper is organised as follows. Section 2 presents configuration and dynamics modeling of the helicopter system. In section 3, controller techniques of helicopter hybrid control system are introduced. Tuning method is described in section 4. Section 5 introduces helicopter control system design is presented. In section 6 simulation results of the hybrid control system are introduced and followed by conclusions and prospective research in section 7.

\section{STRUCTURE AND DYNAMICS MODELING OF HELICOPTER}

\subsection{Helicopter stucture}

The conceptual platform of 3-DOF helicopter system is shown in Figure 1. It consists of an arm mounted on a base. The main body of the helicopter composed of propellers driven by two motors mounted are the either ends of an short balance bar. The whole helicopter body is fixed on one end of the arm and a balance block installed at the other end. The balance arm can rotate about travel axis as well as slope on an elevation axis. The body of helicopter is free to roll about the pitch axis. The system is provided by encoders mounted on these axis used to measure the travel motion of the arm and its elevation and pitch angle. The propellers with motors can generate an elevation mechanical force proportional to the voltage power supplied to the motors. This force can cause the helicopter body to lift off the ground. It is worth considering that the purpose of using balance block is to reduce the voltage power supplied to the propellers motors.

\subsection{Helicopter dynamics and mathematical modeling}

In this study, the nonlinear dynamics of 3DOF helicopter system is modeled mathematically based on developing the model of the system behavior for elevation, pitch and travel axis. The definition of the symbols and nomecalture of the proposed helicopter system is included in Table 1.

\subsubsection{Elevation axis model}

The free body diagram of 3DOF helicopter system based on elevation axis is shown in Figure 2. The movement of the elevation axis is governed by the following differential equations:

$$
J_{\epsilon} \ddot{\epsilon}=M_{c}+l_{m} F_{m} \cos (\rho)-M_{w, \epsilon}-M_{f, \epsilon}
$$

where $M_{c}$ is the centrifugal torque, which is function of $(\epsilon), M_{f, \epsilon}$ is friction moment exerted on the helicopter elevation axis, which is generated from combining joint friction and air resistance, and $M_{w, \epsilon}$ is the effective gravitational torque due to the mass differential $W=m_{h} g$ about the elevation axis. 


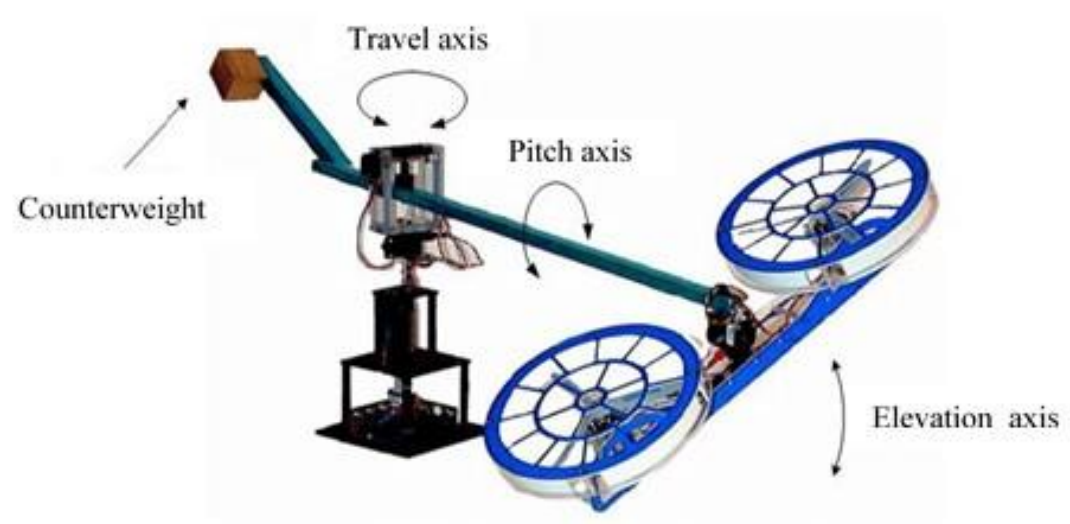

Figure 1. 3DOF Helicopter System [20]

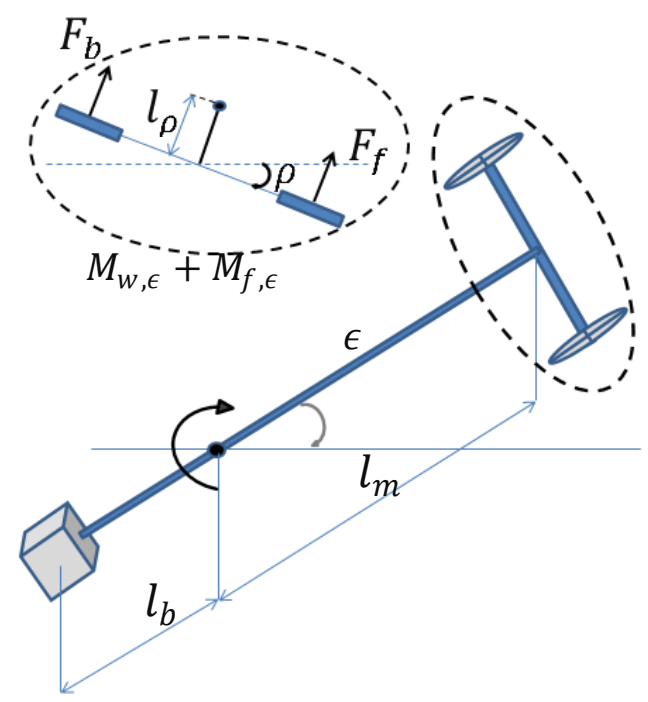

Figure 2. Schematic diagram of elevation axis model for 3DOF helicopter system

$$
M_{w, \epsilon}=l_{m} m_{h} g
$$

If the elevation angle $\epsilon=0$ and the rotation angle of pitch axis $\rho=0$, then the centrifugal torque $\left(M_{c}\right)$ will be zero (1) based on Euler's second law becomes as follows:

$$
\begin{aligned}
& J_{\epsilon} \ddot{\epsilon}=l_{m} F_{m}-M_{w, \epsilon}+M_{f, \epsilon} \\
& J_{\epsilon} \ddot{\epsilon}=l_{m}\left(F_{f}+F_{b}\right)-M_{w, \epsilon}+M_{f, \epsilon} \\
& F_{i}=K_{c} V_{i} \quad i=f, b \\
& J_{\epsilon} \ddot{\epsilon}=K_{c} l_{m}\left(V_{f}+V_{b}\right)-M_{w, \epsilon}+M_{f, \epsilon} \\
& J_{\epsilon} \ddot{\epsilon}=K_{c} l_{m} V_{s}-M_{w, \epsilon}+M_{f, \epsilon}
\end{aligned}
$$

\subsubsection{Pitch axis model}

Consider the pitch schematic diagram of the system in Figure 3. It can be seen from the figure that the main torque acting on the system pitch axis is produced from the thrust force generated by the properller motors. When $\rho \neq 0$, the gravitational force will also generate a torque $M_{w, \rho}$ acts on the helicopter pitch axis. 


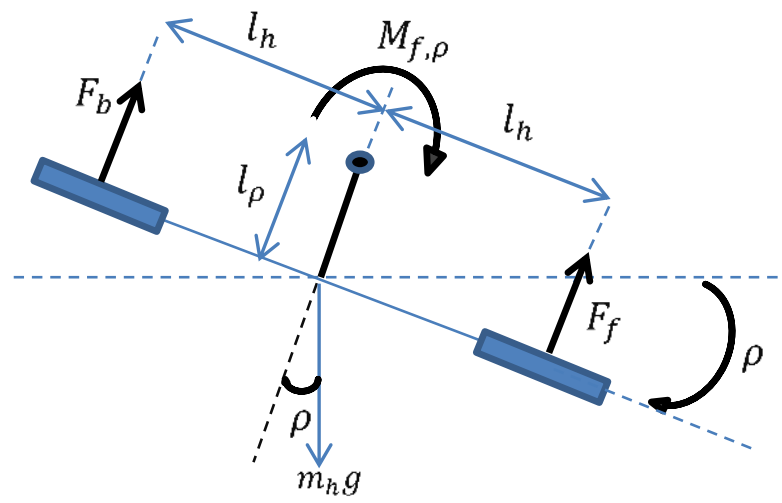

Figure 3. Schematic diagram of pitch axis model for 3DOF helicopter scheme

Table 1. Nomenclature of the helicopter system

\begin{tabular}{cl}
\hline Nomenclature & Definition \\
\hline$\epsilon, \rho, r$ & Rotation angle of elevation, pitch and travel axis respectively (degree) \\
$l_{\rho}$ & Distance between the pitch axis and the helicopter center of gravity (m) \\
$l_{m}$ & Distance from axle point to either propeller motor (m) \\
$l_{b}$ & Distance from axle point to the balance block (m) \\
$l_{h}$ & Distance between the pitch axis and the center of propellers (m) \\
$F_{m}$ & Lift force of the properller motors (N) \\
$F_{f}, F_{b}$ & Forces of fornt and back properller motors (N) \\
$V_{f}, V_{b}$ & Votage applied to the front and back properller motor respectively (v) \\
$m_{b}$ & Mass of balance block (kg) \\
$m_{m}$ & Mass of properller motors (kg) \\
$W$ & Mass differential about the elevation axis (kg) \\
$M_{F, \epsilon}, M_{F, \rho}, M_{F, r}$ & Torque component generated from combining the joint friction and air resistance for elevation, pitch and \\
$M_{c}$ & rate axis respectively (N.m) \\
$M_{w, \epsilon}, M_{w, \rho}$ & Centrifugal torque about the elevation axis (N.m) \\
$j_{\epsilon}, j_{\rho}, j_{r}$ & Effective gravitational torque due to gravity about the elevation axis and pitch axis respectively (N.m) \\
$K_{c}$ & Fortia moment of the system about elevation, pitch and travel axis respectively (kg.m $\left.{ }^{2}\right)$
\end{tabular}

The dynamics of the pitch axis can be modeled mathematically as follows:

$$
J_{\rho} \ddot{\rho}=F_{f} l_{h}-F_{b} l_{h}-M_{w, \rho}-M_{f, \rho}
$$

Where $M_{f, \rho}$ is friction moment exerted on the pitch axis.

$$
M_{w, \rho}=m_{h} g l_{\rho} \sin (\rho) \cos (\epsilon)
$$

Based on the assumption that the pitch angle $\rho=0, M_{w, \rho}=0$, then (8) becomes as follows:

$$
\begin{aligned}
& J_{\rho} \ddot{\rho}=l_{h}\left(F_{f}-F_{b}\right)-M_{f, \rho} \\
& J_{\rho} \ddot{\rho}=K_{c} l_{\rho}\left(V_{f}-V_{b}\right)-M_{f, \rho} \\
& J_{\rho} \ddot{\rho}=K_{c} l_{\rho} V_{d}-M_{f, \rho}
\end{aligned}
$$

\subsubsection{Travel axis model}

The free body diagranm of the helicopter system dynamics based on travel axis is presented in Figure 4. In this model, when $\rho \neq 0$, the main forces acting on the helicopter dynamics are the thrust forces of properller motors $\left(F_{f}, F_{b}\right)$. These forces have a component generates a torque on the travel axis. Assume 
that the helicopter body has roll up by an angle $\rho$ as shown in Figure 4. Then the dynamics of travel axis for 3DOF helicopter system is modeled as follows:

$$
J_{r} \dot{r}=-\left(F_{f}+F_{b}\right) \sin (\rho) l_{m}-M_{f, r}
$$

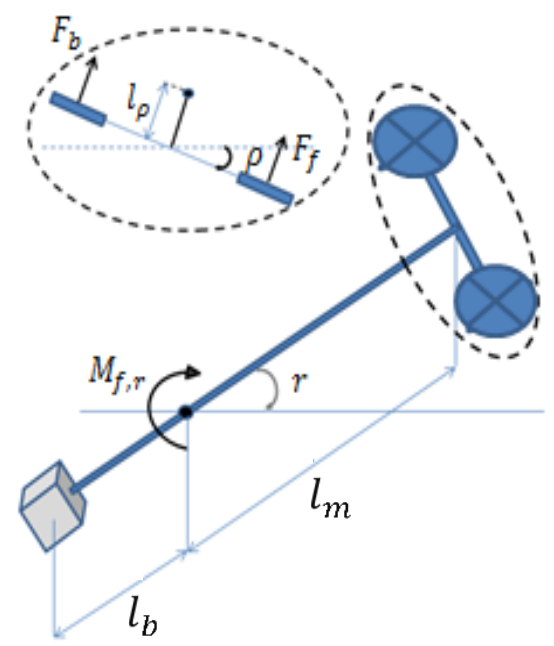

Figure 4. Schematic diagram of travel rate axis model for 3DOF helicopter scheme

The thrust foces of the two properller motors $\left(F_{f}+F_{b}\right)$ are required to keep the helicopter in flight case and is approximately $W$.

$$
J_{r} \dot{r}=-W \sin (\rho) l_{m}-M_{f, r}
$$

Where $M_{f, r}$ is friction moment exerted on travel axis. As $\rho$ approachs to zero, based on sinc function, $\sin (\rho)=\rho$, the above equation becomes as follows:

$$
J_{r} \dot{r}=-W \rho l_{m}-M_{f, r}
$$

Based on the assumption that the coupling dynamics, gravitational torque $\left(M_{w, \epsilon}\right)$ and friction moment exerted on elevation, pitch and travel axis are neglected, then the dynamics modeling (7), (12) and (15) for 3DOF helicopter system can be simplified as in (16), (17) and (18) respectively [1].

$$
\begin{aligned}
& \ddot{\epsilon}=\frac{K_{c} l_{m}}{J_{\epsilon}} V_{s} \\
& \ddot{\rho}=\frac{K_{c} l_{\rho}}{J_{\rho}} V_{d} \\
& \dot{r}=\frac{W l_{m}}{J_{r}} \rho
\end{aligned}
$$

\subsection{System state space model}

In order to design state feedback controller based on LQR technique for 3DOF helicopter system, the dynamics model of the system should be formulated in state space form. In this study, the proposed hybrid control algorithm is investigated for the purpose of control of pitch angle, elevation angle and travel rate of $3 \mathrm{DOF}$ helicopter scheme by regulating the voltage suopplies to the front and back motors. Let $x(\mathrm{nx} 1)=\left[x_{1}, x_{2}, x_{3}, x_{4}, x_{5}, x_{6}, x_{7}\right]^{T}=[\epsilon, \rho, \dot{\epsilon}, \dot{\rho}, r, z, \gamma]^{T}$ be the state vector of the system, the state variables are chosen as the angles and rate and their corresponding angular velocities, and $\dot{z}=\epsilon, \dot{\gamma}=r$. The voltages supplied to the front and back propellers motors are considered the input's vector such that, $u(t)(\operatorname{mx} 1)=\left[u_{1}, u_{2}\right]^{T}=\left[V_{f}, V_{b}\right]^{T}$ and the elevation angle, pitch angle and travel rate are assumed 
the output's vector such that, $y(t)(\mathrm{px} 1)=[\epsilon, \rho, r]^{T}$. Based on (13)-(15), choosing these state variables yields the following system state space model:

$$
\begin{aligned}
& \dot{x_{1}}=\rho=x_{2} \\
& \dot{x_{2}}=\dot{\epsilon}=x_{3} \\
& \dot{x_{3}}=\ddot{\epsilon}=\frac{K_{c} l_{m}}{J_{\epsilon}}\left(V_{f}+V_{b}\right) \\
& \dot{x_{4}}=\ddot{\rho}=\frac{K_{c} l_{\rho}}{J_{\rho}}\left(V_{f}-V_{b}\right) \\
& \dot{x_{5}}=\dot{r}=\frac{W l_{m}}{J_{r}} x_{2} \\
& \dot{x_{6}}=\dot{z}=x_{1} \\
& \dot{x_{7}}=\dot{\gamma}=x_{4}
\end{aligned}
$$

The general state and output matrix equations describing the dynamic behavior of the linear-timeinvariant (LTI) helicopter system in state space form are as follows:

$$
\begin{aligned}
& \dot{x}(t)=A x(t)+B u(t) \\
& y(t)=C x(t)+D u(t)
\end{aligned}
$$

Where $A(\mathrm{nxn})$ is the system matrix, $B(\mathrm{nxm})$ is the input matrix, $C(\mathrm{pxm})$ is the output matrix, and $D(\mathrm{mxp})$ is feed forward matrix, for the designed system. Based on (16), the state space representation (17) and (18) are rewritten as in (19) and (20) [1].

$$
\begin{aligned}
& {\left[\begin{array}{ccccccc}
0 & 0 & 1 & 0 & 0 & 0 & 0 \\
0 & 0 & 0 & 1 & 0 & 0 & 0 \\
0 & 0 & 0 & 0 & 0 & 0 & 0 \\
0 & 0 & 0 & 0 & 0 & 0 & 0 \\
0 & \frac{W l_{m}}{J_{r}} & 0 & 0 & 0 & 0 & 0 \\
1 & 0 & 0 & 0 & 0 & 0 & 0 \\
0 & 0 & 0 & 0 & 1 & 0 & 0
\end{array}\right]\left[\begin{array}{c}
\epsilon \\
\rho \\
\dot{\epsilon} \\
\dot{\rho} \\
r \\
z \\
\gamma
\end{array}\right]+\left[\begin{array}{cc}
0 & 0 \\
0 & 0 \\
\frac{K_{c} l_{m}}{J_{\epsilon}} & \frac{K_{c} l_{m}}{J_{\epsilon}} \\
\frac{K_{c} l_{\rho}}{J_{\rho}} & -\frac{K_{c} \rho}{J_{\rho}} \\
0 & 0 \\
0 & 0 \\
0 & 0
\end{array}\right]\left[\begin{array}{l}
V_{f} \\
V_{b}
\end{array}\right]} \\
& {\left[\begin{array}{l}
\epsilon \\
\rho \\
r
\end{array}\right]=\left[\begin{array}{lllllll}
1 & 0 & 0 & 0 & 0 & 0 & 0 \\
0 & 1 & 0 & 0 & 0 & 0 & 0 \\
0 & 0 & 0 & 0 & 1 & 0 & 0
\end{array}\right]\left[\begin{array}{c}
\epsilon \\
\rho \\
\dot{\epsilon} \\
\dot{\rho} \\
r \\
z \\
\gamma
\end{array}\right]+\left[\begin{array}{ll}
0 & 0 \\
0 & 0 \\
0 & 0
\end{array}\right]\left[\begin{array}{l}
V_{f} \\
V_{b}
\end{array}\right]} \\
& {\left[\begin{array}{c}
\dot{\epsilon} \\
\dot{\rho} \\
\ddot{\epsilon} \\
\ddot{\rho} \\
\dot{r} \\
\dot{\dot{\alpha}} \\
\dot{\gamma}
\end{array}\right]=\left[\begin{array}{ccccccc}
0 & 0 & 1 & 0 & 0 & 0 & 0 \\
0 & 0 & 0 & 1 & 0 & 0 & 0 \\
0 & 0 & 0 & 0 & 0 & 0 & 0 \\
0 & 0 & 0 & 0 & 0 & 0 & 0 \\
0 & 2.0655 & 0 & 0 & 0 & 0 & 0 \\
1 & 0 & 0 & 0 & 0 & 0 & 0 \\
0 & 0 & 0 & 0 & 1 & 0 & 0
\end{array}\right]\left[\begin{array}{c}
\epsilon \\
\rho \\
\dot{\epsilon} \\
\dot{\rho} \\
r \\
z \\
\gamma
\end{array}\right]+\left[\begin{array}{cc}
0 & 0 \\
0 & 0 \\
5.8197 & 5.8197 \\
63.9498 & -63.9498 \\
0 & 0 \\
0 & 0 \\
0 & 0
\end{array}\right]\left[\begin{array}{l}
V_{f} \\
V_{b}
\end{array}\right]}
\end{aligned}
$$

In this study, for purpose of control system design, the model of the system is formulated in state space form using the physical parameters values stated in Table 2 [1]. Based on (19) and using the parameters values in Table 1, the state equation of the system is given by (21). 
Table 2. Values of physical parameters of 3DOF helicopter system

\begin{tabular}{ccc}
\hline Symbol & Physical Unit & Numerical Values \\
\hline$J_{\epsilon}$ & $\mathrm{kg} \cdot \mathrm{m}^{2}$ & 1.8145 \\
$J_{r}$ & $\mathrm{~kg} \cdot \mathrm{m}^{2}$ & 1.8145 \\
$J_{\rho}$ & $\mathrm{kg} \cdot \mathrm{m}^{2}$ & 0.0319 \\
$\mathrm{~W}$ & $\mathrm{~N}$ & 4.2591 \\
$l_{m}$ & $\mathrm{~m}$ & 0.88 \\
$l_{b}$ & $\mathrm{~m}$ & 0.35 \\
$l_{\rho}$ & $\mathrm{m}$ & 0.17 \\
$K_{c}$ & $\mathrm{~N} / \mathrm{V}$ & 12 \\
\hline
\end{tabular}

\section{CONTROLLER TECHNIQUES}

In this section, the theory of the PID and LQR controller techniques used for designing the proposed helicopter control system are introduced. The gain parameters of the PID controller are obtained approximately from the LQR gain matrix. Control equations of elevation angle, pitch angle, and travel rate for helicopter system using PID controller are also presented.

\subsection{PID controller}

A PID is the most popular controller technique that is widely used in industrial applications due to the simplicity of its structure and can be realized easily for various control problems as the gain parameters of the controller are relatively independent [21][22]. Basically, the controller provides control command signals $u(t)$ based on the error $e(t)$ between the demand input and the actual output of the system. The continuous time structure of the PID controller is as follows:

$$
u(t)=K_{p} e(t)+K_{i} \int_{0}^{t} e(\tau) d \tau+K_{d} \frac{d e(t)}{d t}
$$

Where $K_{p}, K_{i}$ and $K_{d}$ are the proportional, integral and differential components of the controller gain. These controller gain parameters should be tuned properly to enable the output states of the system to efficiently follow the desired input. If $\epsilon_{d}, \rho_{d}$ and $r_{d}$ are the desired elevation angle, pitch angle and travel rate of the helicopter system, it can express the form of PID controllers used to meet the desired output states as follows [1,23]. In this study, for elevation angle, the control equation is based on the following PID control equation:

$$
\begin{aligned}
& V_{s}=K_{\epsilon p} e_{\epsilon}+K_{\epsilon d} \dot{e_{\epsilon}}+K_{\epsilon i} \int e_{\epsilon} d t \\
& V_{s}=K_{\epsilon p}\left(\epsilon_{d}-\epsilon\right)-K_{\epsilon d} \dot{\epsilon}+K_{\epsilon i} \int\left(\epsilon_{d}-\epsilon\right) d t
\end{aligned}
$$

While the pitch angle is controlled by the following PD control equation:

$$
\begin{aligned}
& V_{d}=K_{\rho p} e_{\rho}+K_{\rho d} \dot{e_{\rho}} \\
& V_{d}=K_{\rho p}\left(\rho_{d}-\rho\right)-K_{\rho d} \dot{\rho}
\end{aligned}
$$

The travel rate is governed by the following PI control equation:

$$
\begin{aligned}
& \rho_{d}=K_{r p} e_{r}+K_{r i} \int e_{r} d t \\
& \rho_{d}=K_{r p}\left(r_{d}-r\right)+K_{r i} \int\left(r_{d}-r\right) d t
\end{aligned}
$$

where $e_{\epsilon}=\epsilon_{d}-\epsilon, e_{\rho}=\rho_{d}-\rho, e_{r}=r_{d}-r, \dot{e}_{\epsilon}=-\dot{\epsilon}$ and $\dot{e}_{\rho}=-\dot{\rho}$.

\section{2. $L Q R$ controller}

Linear quadratic regulator is a common optimal control technique which has been widely utilized in various manipulator systems [24]. Using LQR technique in design control system requires all the states of the system plant to be measurable as it bases on the full state feedback concept. Therefore, using LQR controller to stabilize the 3DOF helicopter system based on the assumption that the states of the system are considered measurable. LQR approach includes applying the optimal control effort: 


$$
u(t)=-K x(t)
$$

Where $K$ is the state feedback gain matrix of LQR controller, that will enable the output states of the system to follow the trajectories of reference input, while minimizing the following the cost function:

$$
J=\int_{0}^{\infty}\left(x^{T}(t) Q x(t)-u^{T}(t) R u(t)\right) d t
$$

Where $\mathrm{Q}$ and $\mathrm{R}$ are referred to as weighting state and control matrices. The controller feedback gain matrix can be determined by using below equation:

$$
K=R^{-1} B^{T} P
$$

Where $P$ is (nxn) matrix determined from the solution of the following Riccati matrix equation:

$$
A^{T} P+P A-P B R^{-1} B^{T} P+Q=0
$$

In this application, $K=\left[\begin{array}{lllllll}\mathrm{k}_{11} & \mathrm{k}_{12} & \mathrm{k}_{13} & \mathrm{k}_{14} & \mathrm{k}_{15} & \mathrm{k}_{16} & \mathrm{k}_{17} \\ \mathrm{k}_{21} & \mathrm{k}_{22} & \mathrm{k}_{23} & \mathrm{k}_{24} & \mathrm{k}_{25} & \mathrm{k}_{26} & \mathrm{k}_{27}\end{array}\right]$ and $u(t)=\left[\begin{array}{l}u_{1} \\ u_{2}\end{array}\right]$. Based on this, the control effort of the system stated in (26) can be written as follows:

$$
\left[\begin{array}{l}
u_{1} \\
u_{2}
\end{array}\right]=-\left[\begin{array}{lllllll}
\mathrm{k}_{11} & \mathrm{k}_{12} & \mathrm{k}_{13} & \mathrm{k}_{14} & \mathrm{k}_{15} & \mathrm{k}_{16} & \mathrm{k}_{17} \\
\mathrm{k}_{21} & \mathrm{k}_{22} & \mathrm{k}_{23} & \mathrm{k}_{24} & \mathrm{k}_{25} & \mathrm{k}_{26} & \mathrm{k}_{27}
\end{array}\right]\left[\begin{array}{c}
\epsilon \\
\rho \\
\dot{\epsilon} \\
\dot{\rho} \\
r \\
z \\
\gamma
\end{array}\right]
$$

For the purpose of simplicity of control problem the weighting matrices $\mathrm{Q}$ and $\mathrm{R}$ are chosen as the diagonal matrices so that the cost function (27) can be reformulated as below:

$J=\int_{0}^{\infty}\left(q_{11} x_{1}^{2}+q_{22} x_{2}^{2}+q_{33} x_{3}^{2}+q_{44} x_{4}^{2}+q_{55} x_{5}^{2}+q_{66} x_{6}^{2}+q_{77} x_{7}^{2}+r_{11} u_{1}^{2}+r_{22} u_{2}^{2}\right) d t$

Where $q_{11}, q_{22}, q_{33}, q_{44}, q_{55}, q_{66}$ and $q_{77}$ denote the weighting elements of flight angles and their corresponding angular velocities of the proposed 3DOF helicopter system respectively, while, $r_{11}$ and $r_{22}$ are the weighting elements of control inputs. It is worth considering that, in this study, based on the state and control LQR weighting matrices $\mathrm{Q}$ and $\mathrm{R}$, the feedback gain matrix $(K)$ can be calculated by utilizing the Matlab command "lqr".

The optimal control approach LQR is highly recommended for stabilizing the 3DOF helicopter system as it basically looks for a compromising between the best control performance and minimum control effort. Based on LQR controller, an optimum tracking performance can be investigated by a proper setting of the feedback controller gain matrix. To achieve this, the LQR controller is optimized by using GA tuning method which is adopted to obtain an optimum elements values for $\mathrm{Q}$ and $\mathrm{R}$ weighting matrices.

\section{TUNING METHOD}

In this study, GA tuning approach is adopted to optimize the LQR gain matrix used to approximate the gain parameters of PID controller for 3DOF helicopter system. GA is a global search optimization technique bases on the strategy of genetics and natural selection [25][26]. This optimization method is utilized to obtain an optimum global solution for more control and manipulating problems. The procedure of GA approach includes of three basic stepes: Selection, Crossover and Mutation. Applying these main operations creates new individuals which could be better than their parents. Based on the requirements of desired response, the sequence of GA optimization technique is repeated for many iterations and finally stops at generating optimum solution elements for the application problems [27]. The sequence of the GA tuning method is presented in Figure 5 [3]. The steps of the GA loop are defined as follows:

1. Initial set of population.

2. Selecting individuals for mating.

3. Mating the population to create progeny.

4. Mutate progeny. 
5. Inserting new generated individuals into population.

6. Are the system fitness function satisfied?

7. End search process for solution.

In this study, the aim of using GA optimization method is to tune the PID controller parameters by minimizing a selected fitness function which will be discussed in detail in the section 4 . The implementation procedure of the GA tuning method begins with the definition step of the chromosome representation. Each chromosome is represented by nine cells which correspond to the weight matrices elements of the LQR controller as shown in Figure 6. By this representation it can adjust the LQR elements in order to achieve the required performance. These cells are formed by real positive numbers and characterize the individual to be evaluated [27].

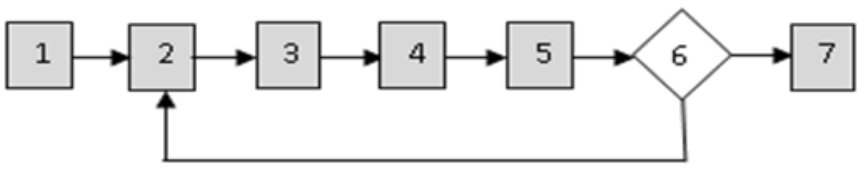

Figure 5. Process loop of GA.

\begin{tabular}{|l|l|l|l|l|l|l|l|l|}
\hline$q_{11}$ & $q_{22}$ & $q_{33}$ & $q_{44}$ & $q_{55}$ & $q_{66}$ & $q_{77}$ & $r_{11}$ & $r_{22}$ \\
\hline
\end{tabular}

Figure 6. Definition of GA chromosome

\section{HELICOPTER HYBRID CONTROLS YSTEM DESIGN}

In this study, a control system using LQR based PID controller is designed to stabilize 3DOF helicopter system. Based on step input, a hybrid controller is designed for the following desired performance parameters: rise time $\left(t_{r}\right)$ less than $10(\mathrm{~ms})$, settling time $\left(t_{s}\right)$ less than $30(\mathrm{~ms})$, maximum overshoot percentage, $\left(M_{O}\right)$ less than $5 \%$. The block diagram of the proposed helicopter control system based on LQR controller is presented in Figure 7.

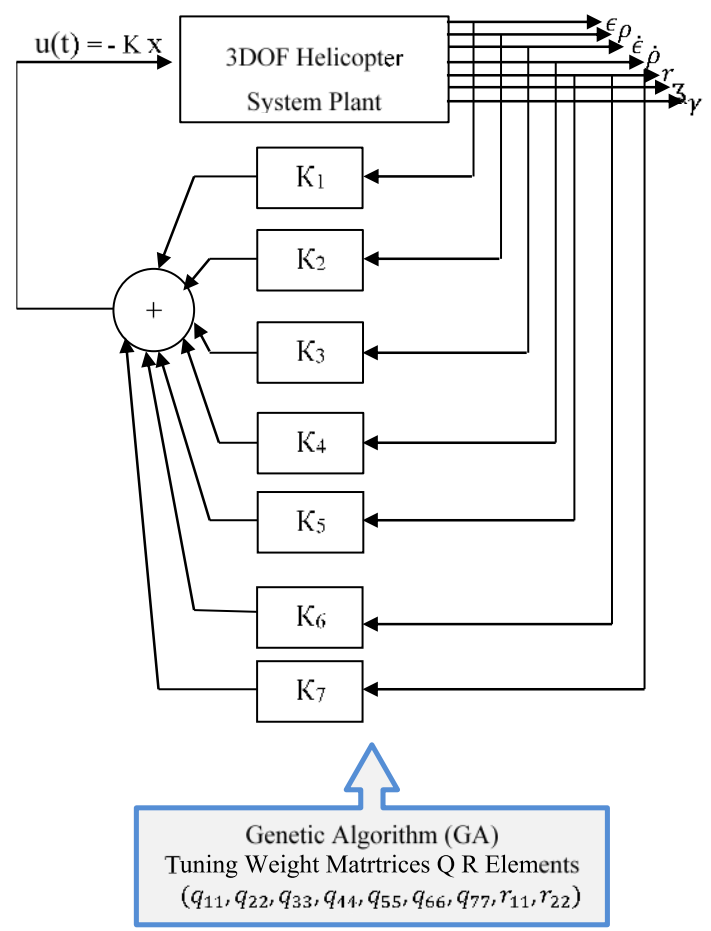

Figure 7. LQR controller based on GA for 3DOF helicopter system 
The control system is analysed mathematically and then simulated using Matlab/simulink tool to validate the proposed LQR controller. Based on the desired performance parameters which include rise and settling time, overshoot and error steady state parameters the fitness function of the control problem is formulated as follows:

$$
F=0.3 S . t_{r}+0.3 S . t_{s}+0.2 S . O+0.2 S . e s S
$$

Where, $S$ is closed loop transfer function of the helicopter system, $S . t_{r}, t_{s}, O, e_{s s}$ are the rise time, settling time, maximum over shoot and error steady state of the closed-loop control system. It is worth considering that the control input effort is considered in the evaluation process of the proposed stabilizing helicopter system. In this study, the design of controller is effectively optimized by using GA tuning method which is adopted to obtain optimum elements values for LQR weighting matrices Q and R. These optimized matrices are using to calcutate optimum controller gain matrix by using (29) and (30). However, the gain matrix is determined by using the Matlab command ' $l q r$ '.

\subsection{PID approximation}

In this subsection, the gain parameters $K_{p}, K_{i}, K_{d}$ of the PID controller are calculated approximately from the feedback gain matrix $K$ of the LQR controller based on GA optimization technique. Analyzing (30) yields the following [4]:

$$
\left[\begin{array}{l}
u_{1} \\
u_{2}
\end{array}\right]=-\left[\begin{array}{ccccccc}
\mathrm{k}_{11} & \mathrm{k}_{12} & \mathrm{k}_{13} & \mathrm{k}_{14} & \mathrm{k}_{15} & \mathrm{k}_{16} & \mathrm{k}_{17} \\
\mathrm{k}_{11} & -\mathrm{k}_{12} & \mathrm{k}_{13} & -\mathrm{k}_{14} & -\mathrm{k}_{15} & \mathrm{k}_{16} & -\mathrm{k}_{17}
\end{array}\right]\left[\begin{array}{c}
\epsilon \\
\rho \\
\dot{\epsilon} \\
\dot{\rho} \\
r \\
z \\
\gamma
\end{array}\right]
$$

\subsubsection{Elevation control using PID controller}

Summing the rows of (33) results the following [1]:

$$
u_{1}+u_{2}=-\left(2 k_{11} \epsilon+2 k_{13} \dot{\epsilon}+2 k_{16} z\right)=-\left(2 k_{11} \epsilon+2 k_{13} \dot{\epsilon}+2 k_{16} \int \epsilon d t\right)
$$

The above equation can be written as

$$
V_{s}=-2 k_{11}\left(\epsilon_{d}-\epsilon\right)-2 k_{13} \dot{\epsilon}-2 k_{16} \int\left(\epsilon_{d}-\epsilon\right) d t
$$

It is obvious that (23b) and (35) have the same structure, this means that the gain parameters of pitch PID controller can be obtained from the gain elements of LQR controller. Thus, comparing (23b) with (35), yields the following gain relationships:

$$
\begin{aligned}
K_{\epsilon p} & =2 k_{11} \\
K_{\epsilon d} & =2 k_{13} \\
K_{\epsilon i} & =2 k_{16}
\end{aligned}
$$

The block diagram of closed-loop control system for 3DOF helicopter system based on PID controller is shown in Figure 8. Taking Laplace transform for elevation axis model (13) yields the following equation:

$$
J_{e} \epsilon(s) \cdot s^{2}=K_{c} l_{1} V_{s}(s)
$$

The transfer function of the elevation axis plant is given by:

$$
\frac{\epsilon(s)}{V_{S}(s)}=\frac{K_{c} l_{m}}{J_{\epsilon} s^{2}}
$$

The transfer function of the PID controller is as follows:

$$
\frac{V_{S}(s)}{E_{\epsilon}(s)}=\frac{K_{\epsilon d} S^{2}+K_{\epsilon p} s+K_{\epsilon i}}{s}
$$


where $E_{\epsilon}(s)=\epsilon_{d}(s)-\epsilon(s)$, the open loop transfer function of the elevation axis control $G_{\epsilon}(s)$ is given by:

$$
G_{\epsilon}(s)=\frac{\epsilon(s)}{E_{\epsilon}(s)}=\frac{V_{S}(s)}{E_{\epsilon}(s)} \frac{\epsilon(s)}{V_{S}(s)}
$$

Based on (38) and (39), the open loop elevation transfer function becomes:

$$
G_{\epsilon}(s)=\frac{K_{c} l_{m}}{J_{\epsilon} s^{2}} \frac{K_{\epsilon d} s^{2}+K_{\epsilon p} s+K_{\epsilon i}}{s}
$$

The closed loop transfer function for elevation angle control is as follows:

$$
\frac{\epsilon(s)}{\epsilon_{c}(s)}=\frac{G_{\epsilon}(s)}{1+G_{\epsilon}(s)}=\frac{K_{c} l_{m} K_{\epsilon d} s^{2}+K_{c} l_{m} K_{\epsilon p} s+K_{c} l_{m} K_{\epsilon i}}{J_{e} s^{3}+K_{c} l_{m} K_{\epsilon d} s^{2}+K_{c} l_{m} K_{\epsilon p} s+K_{c} l_{m} K_{\epsilon i}}
$$

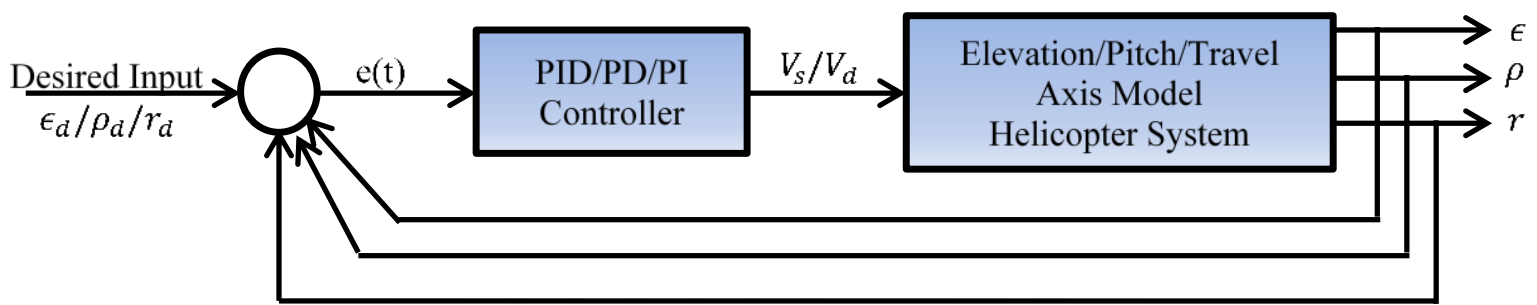

Figure 8. Control system block diagram for helicopter elevation, pitch and travel axis using PID controller

\subsubsection{Pitch control using PD controller}

Similarly, the difference of the rows of (33) results in

$$
\begin{aligned}
& u_{1}-u_{2}=-2 k_{12} \rho-2 k_{14} \dot{\rho}-2 k_{15}\left(r-r_{c}\right)-2 k_{17} \gamma \\
& V_{d}=-2 k_{12} \rho-2 k_{14} \dot{\rho}-2 k_{15}\left(r-r_{d}\right)-2 k_{17} \int\left(r-r_{d}\right) d t
\end{aligned}
$$

Substitution $(25 b)$ in $(24 b)$ results,

$$
V_{d}=-K_{\rho p} \rho-K_{\rho d} \dot{\rho}+K_{\rho p} K_{r p}\left(r_{d}-r\right)+K_{\rho p} K_{r i} \int\left(r_{d}-r\right) d t
$$

It is clear that (44) and (45) have exactly the same structure. Then, by comparing these equations, it can obtain the feedback gains parameters for the PID controller from the LQR gains parameters as follows:

$$
\begin{gathered}
K_{\rho p}=2 k_{12} \\
K_{\rho d}=2 k_{14} \\
K_{r p}=\frac{k_{15}}{k_{12}} \\
K_{r i}=\frac{k_{17}}{k_{12}}
\end{gathered}
$$

Taking Laplace transform for pitch axis model (14) yields:

$$
J_{\rho} \rho(s) s^{2}=K_{c} l_{\rho} V_{d}(s)
$$

The transfer function for pitch axis model is given by:

$$
\frac{\rho(\mathrm{s})}{\mathrm{V}_{\mathrm{d}}(\mathrm{s})}=\frac{K_{c} l_{\rho}}{J_{\rho} \mathrm{s}^{2}}
$$


The transfer function of the PD controller is as follows:

$$
\frac{V_{d}(s)}{E_{\rho}(s)}=K_{\rho d} s+K_{\rho p}
$$

where $E_{\rho}(s)=\rho_{d}(s)-\rho(s)$, the open loop transfer function of the pitch axis control is given by:

$$
G_{p}(s)=\frac{\rho(s)}{E_{\rho}(s)}=\frac{\rho(s)}{V_{d}(s)} \frac{V_{d}(s)}{E_{\rho}(s)}=\frac{K_{c} l_{\rho}\left(K_{\rho d} s+K_{\rho p}\right)}{J_{\rho} s^{2}}
$$

The closed loop transfer function of pitch angle is given by:

$$
\frac{\rho(s)}{\rho_{c}(s)}=-\frac{K_{c} l_{\rho} K_{\rho d} s+K_{c} l_{\rho} K_{\rho p}}{J s^{2}+K_{c} l_{\rho} K_{\rho d}+K_{c} l_{\rho} K_{\rho p}}
$$

\subsubsection{Travel control using PI controller}

Taking Laplace transform for travel axis model (15) results:

$$
r(s) s=\frac{W l_{m}}{J_{r}} \rho(s)
$$

The transfer function for travel axis model is given by:

$$
\frac{\mathrm{r}(\mathrm{s})}{\rho(\mathrm{s})}=\frac{W l_{m}}{J_{r} \mathrm{~s}}
$$

The transfer function of the PI controller is as follows:

$$
\frac{\rho(s)}{E_{r}(s)}=K_{r p}+\frac{K_{r i}}{s}
$$

where $E(s)=\rho_{d}(s)-\rho(s)$, the open loop transfer function of the travel axis control is given by:

$$
G_{r}(s)=\frac{\mathrm{r}(\mathrm{s})}{\rho(\mathrm{s})} \frac{\rho_{\rho}(s)}{E_{r}(s)}=\frac{W l_{m}\left(K_{r p} s+K_{r i}\right)}{J_{r} s^{2}}
$$

The closed loop transfer function for travel angle is as follows:

$$
\frac{\mathrm{r}(\mathrm{s})}{\mathrm{r}_{\mathrm{d}}(\mathrm{s})}=\frac{W l_{m} K_{r p} s+W l_{m} K_{r i}}{J_{r} s^{2}+W l_{m} K_{r p} s+W l_{m} K_{r i}}
$$

\section{CONTROLLER SIMULATION AND RESULTS}

\subsection{LQR controller}

In order to validate the proposed helicopter stabilizing system, the LQR controller is analysed mathematically using Matlab environment. Based on objective function ( $\mathrm{J}$ ) and using the Matlab command "lqr" the elements of the LQR weighting matrices Q, R are tuned using GA optimization method. The parameters of the GA optimization approach chosen for tuning process of the 3DOF helicopter control system are listed in Table 3. Converging elements of the LQR weighting matrices $\mathrm{Q}, \mathrm{R}$ through iteration based on GA optimization method are presented in Figure 9.

Table 3. Parameters of GA tuning method

\begin{tabular}{ll}
\hline GA Parameter & Value/Method \\
\hline Population Size & 20 \\
Max No. of Generations & 100 \\
Selection Method & Normalized Geometric Selection \\
Crossover Method & Scattering \\
Mutation Method & Uniform Mutation \\
\hline
\end{tabular}



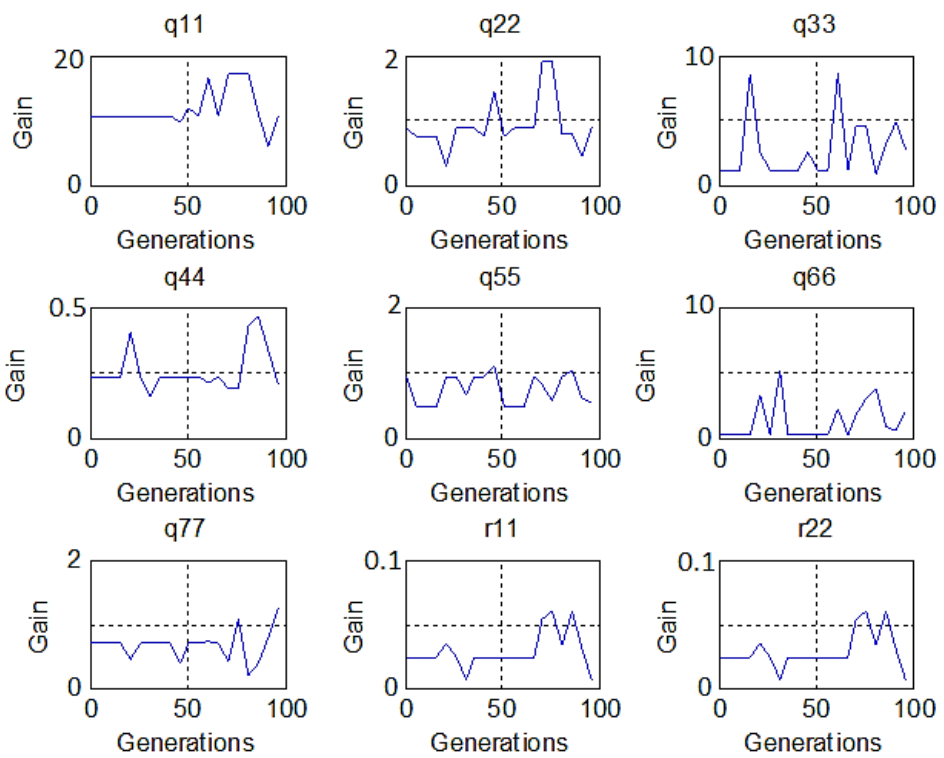

Figure 9. Number of generation of GA-LQR parameters $Q$ and $R$

Based on the fitness function, the optimal elements values for LQR weighting matrices $\mathrm{Q}, \mathrm{R}$ obtained based on the GA tuning approach are given by:

$$
Q=\left[\begin{array}{ccccccc}
9.0425 & 0 & 0 & 0 & 0 & 0 & 0 \\
0 & 0.7840 & 0 & 0 & 0 & 0 & 0 \\
0 & 0 & 4.5093 & 0 & 0 & 0 & 0 \\
0 & 0 & 0 & 0.2763 & 0 & 0 & 0 \\
0 & 0 & 0 & 0 & 0.7077 & 0 & 0 \\
0 & 0 & 0 & 0 & 0 & 0.9876 & 0 \\
0 & 0 & 0 & 0 & 0 & 0 & 0.8004
\end{array}\right], R=\left[\begin{array}{cc}
0.0483 & 0 \\
0 & 0.0483
\end{array}\right]
$$

By using the state matrix $(A)$, input matrix $(B)$, and weighting matrices $(Q, R)$, the optimized feedback gain matrix $K$ and closed-loop rictions of the system are determined using the Matlab command "lqr". The calculated gain matrix $K$ is given by:

$$
K=\left[\begin{array}{ccccccc}
117.138 & 65.9975 & 68.4252 & 16.9323 & 50.6954 & 31.954 & 28.766 \\
117.138 & -65.9975 & 68.4252 & -16.9323 & -50.6954 & 31.954 & -28.766
\end{array}\right]
$$

The poles of the closed-loop control system for helicopter using optimized GA-LQR controller are listed in Table 4. Based on the feedback gain matrix and using (26), the LQR control effort vector for the 3DOF helicopter system is as follows:

Table 4. Poles of helicopter control system based on LQR technique

\begin{tabular}{cc}
\hline Pole & Value \\
\hline$P_{1}$ & -794.7130 \\
$P_{2}$ & -1.3745 \\
$P_{3}$ & -0.3405 \\
$P_{4}$ & -2161.7 \\
$P_{5}$ & $-1.3623+\mathrm{i} 1.0631$ \\
$P_{6}$ & $-1.3623-1.0631 \mathrm{i}$ \\
$P_{7}$ & -1.1773 \\
\hline
\end{tabular}

$$
\left[\begin{array}{l}
u_{1} \\
u_{2}
\end{array}\right]=-\left[\begin{array}{ccccccc}
117.138 & 65.998 & 68.425 & 16.932 & 50.695 & 31.954 & 28.766 \\
117.138 & -65.998 & 68.425 & -16.932 & -50.695 & 31.954 & -28.766
\end{array}\right]\left[\begin{array}{c}
\epsilon \\
\rho \\
\dot{\epsilon} \\
\dot{\rho} \\
r \\
z \\
\gamma
\end{array}\right]
$$




\subsection{PID controller}

Based on (36), (46) and (57), the absolute values of PID, PD and PI gain parameters for elevation, pitch and travel axis model respectively for helicopter system are listed in Table 5 [1]. Using the values in Table 2 and 5, the closed-loop transfer function of elevation, pitch and travel axis (42), (51) and (56) become as in (58), (59) and (60) respectively:

Table 5. Values of gain parameters for PID, PD and PI controllers

\begin{tabular}{ccc}
\hline PID Parameter & Relationship & Absolute Value \\
\hline$K_{\epsilon p}$ & $2 k_{11}$ & 234.2765 \\
$K_{\epsilon d}$ & $2 k_{13}$ & 136.8504 \\
$K_{\epsilon i}$ & $2 k_{16}$ & 63.9086 \\
$K_{\rho p}$ & $2 k_{12}$ & 131.9949 \\
$K_{\rho d}$ & $2 k_{14}$ & 33.8645 \\
$K_{r p}$ & $k_{15} / k_{12}$ & 0.7681 \\
$K_{r i}$ & $k_{17} / k_{12}$ & 0.4359 \\
\hline
\end{tabular}

$$
\begin{aligned}
\frac{\epsilon(s)}{\epsilon_{c}(s)} & =\frac{1445 s^{2}+247 s+674.9}{1.8145 s^{3}+1445 s^{2}+2474 s+674.9} \\
\frac{\rho(s)}{\rho_{c}(s)} & =\frac{69.08 s+269.3}{0.0319 s^{2}+69.08 s+269.3} \\
\frac{r(s)}{r_{c}(s)} & =\frac{2.878 s+1.634}{1.815 s^{2}+2.878 s+1.634}
\end{aligned}
$$

Based on bounded input signal, the elevation, pitch and travel axis model of 3DOF helicopter system are unstable as they give unbounded outputs. The output time responses for elevation, pitch and travel angle are illustrated in the Figure 10. In this study, in order to to achieve a stable output, a hybrid control system using LQR based PID controller for 3DOF helicopter system is proposed to control the dynamic behavior of the system. To validate the proposed helicopter stabilizing scheme, the controller is simulated using Matlab programming tool. Three axis, elevation, pitch, travel rate, are considered in the simulation process of the control system. The performance of the helicopter balancing system is evaluated under unit step reference input using rise, settling time, overshoot and steady state error parameters for the elevation, pitch and travel angles to simulate the desired command given by the pilot.

\subsubsection{Elevation axis model simulation}

This section deals with the simulatin of LQR based PID controller used to control the position of helicopter elevation model. Figure 11 presents tracking control curve of the demand input based on PID controller for helicopter elevation angle. The simulation result shows that the controller succeeded to guide the output state of the system through the desired input trajectory effectively with negligible overshoot, short rise and settling time of $0.1 \mathrm{~ms}$ and $0.3 \mathrm{~ms}$ respectively.

\subsubsection{Pitch axis model simulation}

In this section, GA-LQR based PD controller is designed to control the dynamic model of helicopter pitch angle. Based on the optimized PD gain parameters stated in Table 4, the output response of proposed helicopter tracking system is illustrated in Figure 12. It is obvious that the controller forced the pitch angle state to follow the desired trajectory effectively.

\subsubsection{Travel axis model simulation}

The control of travel rate for 3DOF helicopter system is governed GA-LQR based PI controller. The output response of the PI tracking scheme using optimum gain parameters listed in Table 5 is shown in Figure 13. It can be noted that the optimized hybrid controller enabled the output state to track the desired input trajectory without overshoot, and shorter rise and settling time with minimal steady state tracking error. Regarding the control effort, the curves of input signals supplied to the propeller motors for the proposed 3DOF helicopter system are shown in Figure 14. It can be seen from input response that the control inputs of the helicopter control system were within acceptable values. Based on the Figures 11-13, it can say that the control performance of optimized GA-LQR based PID, PD and PI controllers for helicopter elevation, pitch and travel axis model respectively was acceptable through tracking the system output states for the reference input efficiently. From the mini plots of Figures 11-13, the performance parameters of PID, PD and PI controller for helicopter elevation, pitch and travel axis are listed in the Table 6. The results in the table show the effectiveness of the optimized hybrid controllers for helicopter system application. 


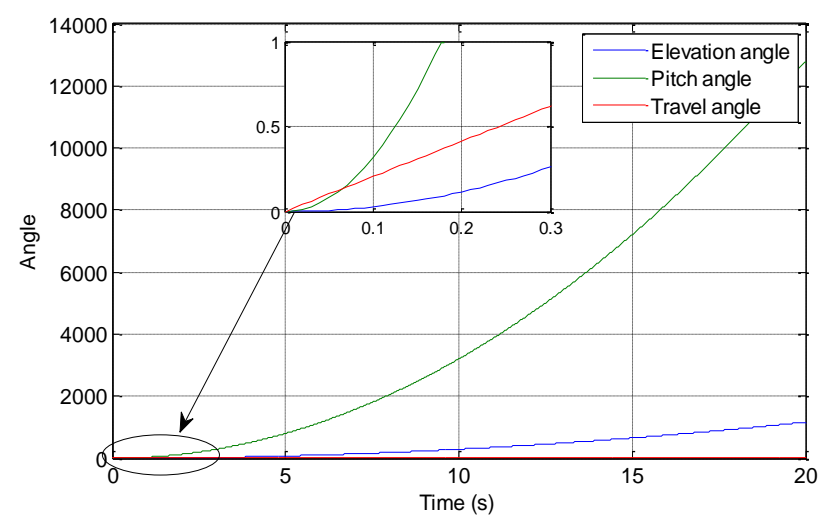

Figure 10. Open-loop response of helicopter system

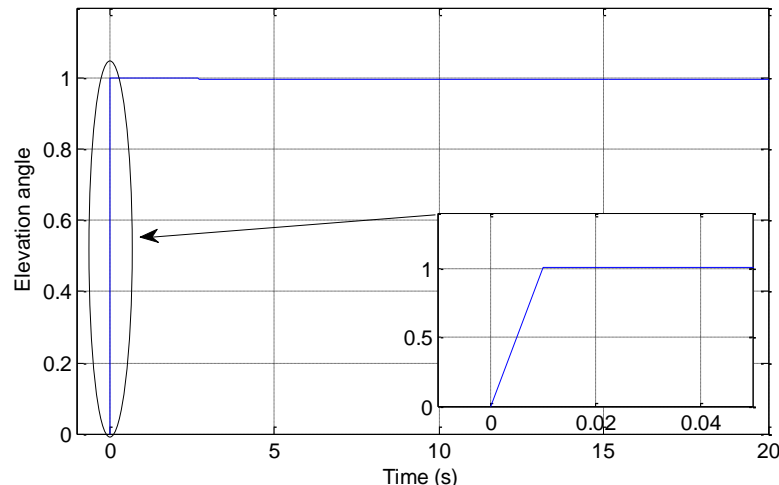

Figure 11. Output response of closed-loop helicopter control system for elevation angle state

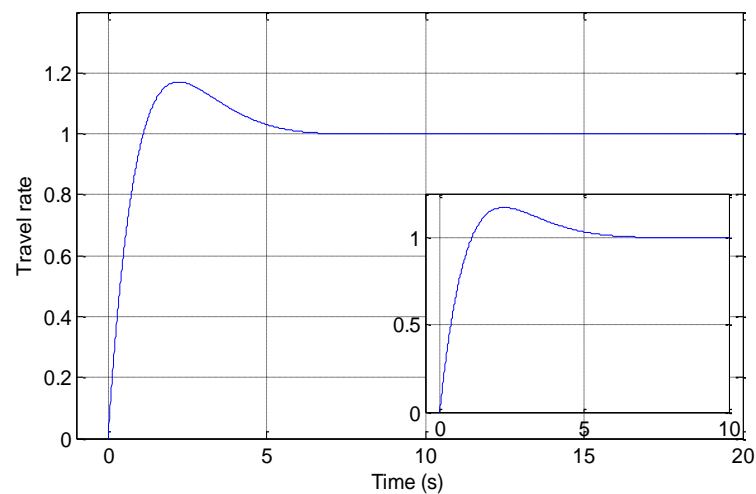

Figure 13. Output response of closed-loop helicopter control system for travel rate state

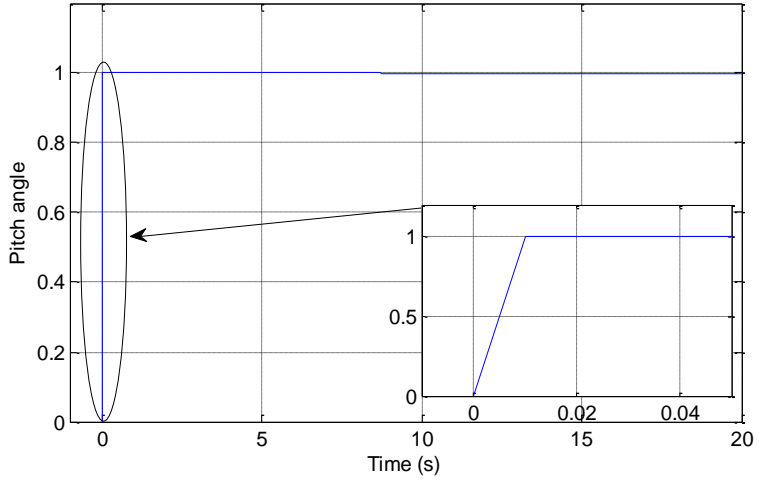

Figure 12. Output response of closed-loop helicopter control system for pitch angle state

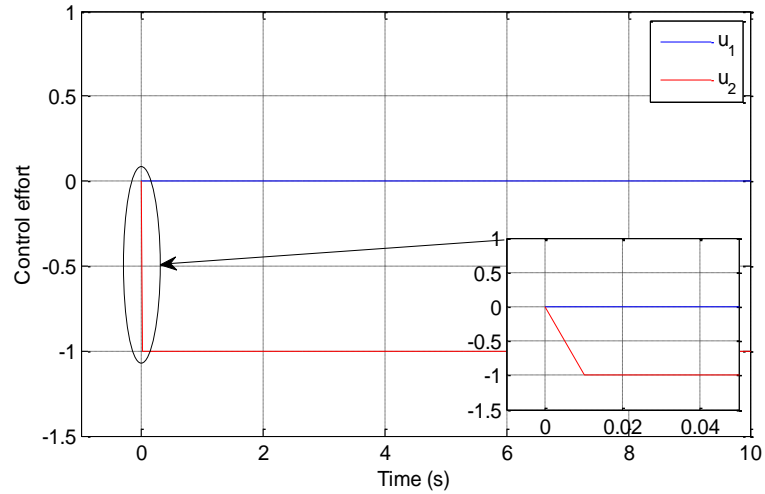

Figure 14. Conrol input of 3DOF helicopter control system

Table 6. Performance parameters of helicopter controller system

\begin{tabular}{cccc}
\hline Controller & $\operatorname{Tr}(\mathrm{sec})$ & $\mathrm{Ts}(\mathrm{sec})$ & $\mathrm{Mp} \%$ \\
\hline Elevation PID & 0.343 & 0.535 & 1.1 \\
Pitch PD & 0.582 & 1.05 & 0 \\
Travel PI & 1.17 & 12.4 & 5.29 \\
\hline
\end{tabular}




\section{CONCLUSION}

In this study, an efficient hybrid control system has been developed for 3DOF helicopter system. The dynamics of elevation, pitch and travel axis for helicopter system is modeled mathematically and then formulated in state space form to enable utilizing state feedback controller technique. In the proposed helicopter stabilizing scheme, a combination of a conventional PID control with LQR state feedback controller is adopted to stabilize the elevation, pitch and travel axis of the helicopter scheme. The gain parameters of the traditional PID controller are determined from the gain matrix of state feedback LQR controller.

In this research, the LQR controller is optimized by using GA tuning technique. The GA optimization method has been adopted to find optimum values for LQR gain matrix elements which are utilized to find best PID gain parameters. The output response of the optimized helicopter control system has been evaluated based on rise time, setting time, overshoot and steady state error parameters. The simulation results have shown the effectiveness of the proposed GA-LQR based PID controller to stabilize the helicopter system at desired values of elevation and pitch angle and travel parameters.

\section{REFERENCES}

[1] S. K., Choudhary, "LQR Based PID Controller Design for 3-DOF Helicopter System," International Journal of Electrical and Information Engineering, vol.8, no.8, pp. 1498-1503, 2014.

[2] S. Franko, "LQR-Based Trajectory Control of Full Envelope, Autonomous Helicopter," World Congress of Engineering 2009, vol. I, London, 1-3, July 2009.

[3] N. Thomas and P. Poongodi, "Position Control of DC Motor Using Genetic Algorithm Based PID Controller," World Congress of Engineering 2009, vol. II, London, 1-3 July, 2009.

[4] L. Fan, E. Joo, "Design for Auto-tuning PID Controller Based on Genetic Algorithms," IEEE $4^{\text {th }}$ International Conference on Industrial Electronics and Applications, 2009

[5] I. Mohammed and A. Ibrahim, "Design of optimised linear quadratic regulator for capsule endoscopes based on artificial bee colony tuning algorithm”, International Journal for Engineering Modeling, vol. 31, no.12, pp. 77-98, 2018.

[6] K. Ogata, Modern Control Engineering. Prentice-Hall International Upper Saddle River, NJ, 1997.

[7] A. Tewari, Modern Control Design with Matlab and Simulink, John Willey and Sons, LTD; 2005.

[8] F. Hasbullah, W. Faris, "A Comparative analysis of LQR and fuzzy logic controller for active suspension using half car model," IEEE international Conference on Control Automation Robotics and Vision, pp. 2415-2420, 2010.

[9] H. Liouane, I. Chiha, A. Douik, and H. Messaoud. "Probabilistic differential evolution for optimal design of lqr weighting matrices," IEEE International Conference on Computational Intelligence for Measurement Systems and Applications (CIMSA), pp. 18-23, 2012.

[10] D. Srinivasan, T. H. Seow, Evolutionary Computation, CEC '03, Canberra, Australia, vol. 4, pp. 2292-2297, 2003.

[11] X. Zeng, L. Jing, Z. Yao, and Y. Guo, "A PSO-based LQR controller for accelerator PWM power supply," $2^{\text {nd }}$ International Conference on Mechatronics and Intelligent Materials, MIM 2012, May 18-19, pp. 71-75, 2012.

[12] M. B. Poodeh, S. Eshtehardiha, A. Kiyoumarsi, and M. Ataei, "Optimizing LQR and pole placement to control buck converter by genetic algorithm," International Conference on Control, Automation and Systems, ICCAS '07, pp.2195-2200, 2007.

[13] J. Zhang, L. Zhang, and J. Xie, "Application of memetic algorithm in control of linear inverted pendulum," IEEE International Conference on Cloud Computing and Intelligence Systems, pp. 103-107, 2011.

[14] E. Rakhshani, "Intelligent linear-quadratic optimal output feedback regulator for a deregulated automatic generation control system, "Electric Power Components and Systems, vol. 40, no. 5, pp. 513-533, 2012.

[15] A. Jacknoon, M. A. Abido, "Ant Colony based LQR and PID tuned parameters for controlling Inverted Pendulum, " IEEE International Conference on Communication, Control, Computing and Electronics Engineering (ICCCCEE), pp. 19-25, 2017.

[16] B. Ata, R. Coban, "Artificial Bee Colony algorithm based Linear Quadratic optimal controller design for a nonliear Inverted Pendulum," International Journal of Intelligent System and Applications in Engineering, vol. 3 no. 1, pp. 1-6, 2015.

[17] S. P. Ramaswamy, G. K Venayagamoorthy, and S. Balakrishnan, "Optimal control of class of non-linear plants using artificial immune systems: Application of the clonal selection algorithm," IEEE 22nd International Symposium on Intelligent Control, pp. 249-254, 2007.

[18] E. V. Kumar and J. Jerome, "LQR based optimal tuning of PID controller for trajectory tracking of Magnetic Levitation System," International Conference On DESIGN AND MANUFACTURING, Procedia engineering 24, pp. 254-264, 2013.

[19] A. N. Khizer, et al., "3DoF Model Helicopter with Hybrid Control," TELKOMNIKA Indonesian Journal of Electrical Engineering, vol.12, no. 5, pp. 3863-3872, 2014.

[20] R. Akmeliawati and S. Raafat, "Optimized State Feedback Regulation of 3DOF Helicopter System via Extremum Seeking," $9^{\text {th }}$ Asian Control Conference, pp. 1-6, 2013.

[21] I. Mohammed, B. Sharif and J. Neasham, "Design and implementation of a magnetic levitation control system for robotically actuated capsule endoscopes," IEEE International Symposium on Robotic and Sensors Environments ROSE2012, pp. 140-145, 2012. 
[22] I. Mohammed, A. Abdulla, "Fractional Order PID Controller Design for Speed Control DC Motor based on Artificial Bee Colony Optimization, ”International Journal of Computer Applications, vol. 179, no. 24, pp. 43-49, 2018.

[23] M. Bharathi, G. Kumar, "Design Approach for Pitch Axis Stabilization Of 3-Dof Helicopter System an LQR Controller," International Journal of Advanced Research in Electrical, Electronics and Instrumentation Engineering, vol. 1, no. 5, pp. 351-365, 2012.

[24] J. Fang, "The LQR Controller Design of Two-Wheeled Self-Balancing Robot Based on the Particle Swarm Optimization Algorithm," Mathematical Problems in Engineering, vol. 2014, pp. 1-6, June 2014.

[25] C. Wongsathan, C. Sirima, "Application of GA to Design LQR Controller for an Inverted Pendulum System," IEEE International Conference on Robotics and Biomimetics, pp. 951-954, 2008.

[26] M. J. Neath et al., "An Optimal PID Controller for a Bidirectional Inductive Power Transfer System Using Multiobjective Genetic Algorithm, " IEEE Transaction on Power Electronic, vol. 29, no. 3 pp. 1523-1531, 2014.

[27] A. Abdulla., I. Mohammed and A. Jasim, "Roll Control System Design Using Auto Tuning LQR Technique," International Journal of ngineering and Innovative Technology, vol.6, no. 2, pp. 11-22. 